\title{
Hybrid Energy-Efficient Clustering Protocols for Wireless Sensor Networks
}

\author{
Shuo Chen \\ School of Information Science and \\ Engineering \\ Shandong University \\ Jinan, P. R. China \\ bitmouse.micky@gmail.com
}

\author{
Maiying Shen \\ School of Information Science and \\ Engineering \\ Shandong University \\ Jinan, P. R. China \\ shenmaiying@126.com
}

\author{
Qi Cao \\ School of Information Science and \\ Engineering \\ Shandong University \\ Jinan, P. R. China \\ 391715743@qq.com
}

\begin{abstract}
Wireless Sensor Networks (WSNs) with numerous spatially distributed sensors can accurately monitor a remote environment, such as humidity, pressure, distance, etc. and cooperatively send the data, combing from the individual nodes, to base station (BS). Energy saving is a great challenge for WSNs which have limited power. Many protocols based on the clustering algorithm are specifically put forward for WSNs, such as LEACH, DEEC, SEP, TEEN, and etc. In this paper, we provide and analyze a new hybrid energy-efficient clustering (HEEC) protocol designed for heterogeneous environment in WSNs. In HEEC, Cluster Head (CH) selection depends on the ratio of surplus energy of every node to average energy of the whole network. Moreover, in order to save and balance energy conservation and suit time-critical applications' requirement, we introduce threshold of detected data by each node. Simulation results and theoretical analysis represent the proposed protocol outperforms DDEEC and TEEN in terms of network lifetime, stability period, data packets received by BS and energy consumption in wireless sensor networks.
\end{abstract} WSNs;

Keywords- clustering algorithm; energy-efficient; hybrid;

\section{INTRODUCTION}

Wireless Sensor Networks which is consisted of numerous low-cost, low-power, multi-function sensor nodes, which usually are placed inside buildings on the ground, in vehicles, or in the air, which can coordinate effectively with each other to perform a given task or transmit the sensed data to BS through wireless communication networks [1]. WSNs has been widely used both in civilian and military application areas, such as environmental monitoring, passive localization and tracking, industrial process control and battlefield surveillance [2]. They are all used in the environment where a fixed infrastructure is impractical or inconvenient. In recent literatures, many protocols and algorithms has been proposed and developed for wired and wireless ad hoc networks, while most of them cannot be directly used in WSNs considering their special and unique structure with limited memory and computational resources and energy storage. Therefore, many clustering algorithms have been particularly constructed for WSNs and their clustering algorithms are quite different due to many factors. Low-Energy Adaptive Clustering Hierarchy (LEACH) [3, 4],
Distributed Energy-Efficient Clustering (DEEC) [5] and Developed DEEC (DDEEC) [6] are representatives of clustering protocols for WSNs [7-8]. In fact, users only focus on the data which reaches the given threshold or the change of which reaches the given threshold in some special time critical applications that need to response immediately in the relevant parameters of interest. In this paper, a new hybrid energy-efficient clustering (HEEC) protocol are provided and analyzed in WSNs, which not only solves this issue but also achieves higher performance in the field of stability period, network lifetime, the number of message packets to BS and energy-saving. The outline of this paper is as follows. A radio model is first provided in Section II. Then in Section III, the details of our proposed protocol in WSNs are presented. Additionally, Section IV gives the discussion, analyze and simulation results. Finally, conclusion of this work is summarized in Section V.

\section{RADIO MODEL}

Similar to LEACH [3], we assume a brief model of energy consumption of radio hardware as shown in Fig. 1.

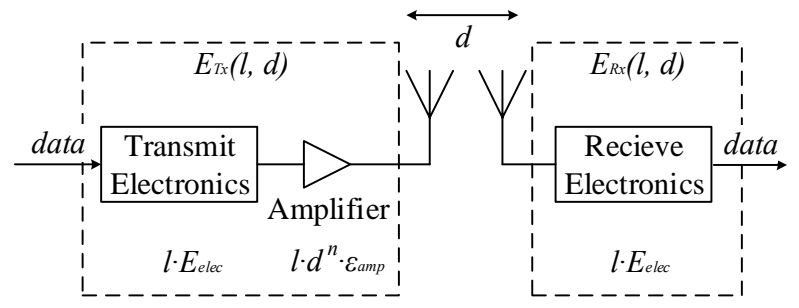

Figure 1. Radio energy dissipation model.

In this model, we use two channel models. If the distance from transmitter to receiver is more than a threshold $d_{0}$, the multipath (mp) model is used, otherwise, the free space (fs) model is used. We assume that an l-bit message is transmitted with a distance $d$, the energy consumes 


$$
\begin{aligned}
E_{T x}(l, d) & =E_{T x-\text { elec }}(l)+E_{T x-a m p}(l, d) \\
& = \begin{cases}l \cdot E_{\text {elec }}+l \cdot \varepsilon_{f s} \cdot d^{2} & d<d_{0}, \\
l \cdot E_{\text {elec }}+l \cdot \varepsilon_{m p} \cdot d^{4} & d \geq d_{0}\end{cases}
\end{aligned}
$$

and the energy consumption of receiving message is

$$
E_{R x}(l)=E_{R x-\text { elec }}(l)=l \cdot E_{\text {elec }},
$$

where the value of $d_{0}$ is

$$
d_{0}=\frac{\varepsilon_{f s}}{\varepsilon_{m p}} .
$$

\section{The Details of Our Proposed Protocol HeEC}

A lot of clustering routing protocols have been designed for WSNs. DDEEC is one of the clustering protocols those are most energy-efficient in heterogeneous networks. However, it is not well suited for some time critical applications or some applications which users only want to pay attentions on one aspect or several aspects, especially in many military applications. TEEN [9] is designed for time critical applications in homogeneous environment and cannot adapt well to heterogeneous environment. Hence, in our protocol HEEC, we focus on both two aspects to improve the stable region for clustering hierarchy process in WSNs.

In HEEC, the $\mathrm{CH}$ selection algorithm is based on LEACH, which make each node $s_{i}(i=1,2, \ldots, N)$ to become a CH in every $n_{i}=1 / P_{i}$ rounds. The probability threshold of $s_{i}$, which is used to decide whether it could be selected as a $\mathrm{CH}$, is defined as

$$
T\left(s_{i}\right)=\left\{\begin{array}{cc}
\frac{P_{i}}{1-P_{i}\left(r \bmod \frac{1}{P_{i}}\right)} & \text { if } s_{i} \in G \\
0 & \text { otherwise }
\end{array},\right.
$$

in which $G$ is a set which includes nodes those are eligible to be $\mathrm{CHs}$ at round $\mathrm{r}$. By computing the reference energy that each node expends during each round and estimating the ideal value of network lifetime, each node requires no global knowledge.

We use the probability of initial and residual energies of a node to select to be a cluster head which is similar to that of DDEEC. In this paper, we consider two kinds of nodes which are defined as advanced nodes with initial energy $E_{0} \cdot(1+a)$ and normal nodes with initial energy $E_{0}$ and the number of them are $m \cdot N$ and $(1-m) \cdot N$ respectively, where $E_{0}$ is the initial energy and $a$ and $m$ are two variables which control the advanced nodes percentage and the initial energy of advanced nodes respectively. The total initial energy of the whole networks is defined by

$$
\begin{aligned}
E_{\text {total }} & =N \cdot(1-m) \cdot E_{0}+N \cdot m \cdot E_{0} \cdot(1+a) . \\
& =N \cdot E_{0} \cdot(1+a \cdot m)
\end{aligned}
$$

The probability of $\mathrm{CH}$ is defined as follows:

$$
P_{i}= \begin{cases}\frac{P_{o p t} \cdot E_{i}(r)}{(1+a \cdot m) \cdot E(r)} & \text { for Normal, } E_{i}(r)>T h_{R E V} \\ \frac{(1+a) \cdot P_{o p t} \cdot E_{i}(r)}{(1+a \cdot m) \cdot E(r)} & \text { for Advance, } E_{i}(r)>T h_{R E V} . \\ c \frac{(1+a) \cdot P_{o p t} \cdot E_{i}(r)}{(1+a \cdot m) \cdot E(r)} & \text { for All, } E_{i}(r)>T h_{R E V}\end{cases}
$$

The value of $T h_{R E V}$ is written as $T h_{R E V}=b E_{0}$ where

$$
b=1+\frac{a \cdot E_{d i s N N}}{E_{d i s N N}-E_{d i s A N}} .
$$

Through numerous simulation with random deployment architecture topology, we find the perfect value of $b$ is 0.7 and perfect value of $c$ is 0.02 , then:

$$
T h_{\text {REV }} \approx 0.7 E_{0} .
$$

Supposing the deployment of nodes conforms to uniform distribution, we can get:

$$
d_{t o C H}=\frac{M}{\sqrt{2 \pi \cdot k}},
$$

$$
d_{t o B S}=0.765 \frac{M}{2} .
$$

where $M$ is the length of distributed region, and the prefect value of $k_{\text {opt }}$ is defined by

$$
k_{o p t}=\frac{\sqrt{N}}{\sqrt{2}} \sqrt{\frac{\varepsilon_{f s}}{\varepsilon_{m p}}} \frac{M}{d_{t o B S}^{2}} .
$$

In order to improve the efficiency of useful data transmission, save the energy conservation and fit for the time critical applications' requirements, we introduce two threshold values that impose restrictions on the transmission of the sensed data. When cluster formation is done, the $\mathrm{CHs}$ transmit one threshold value- absolute threshold (AT) to every node. The node senses the environment repeatedly and 
compares the sensed data with assigned AT value first by itself. If the current value of sensed (CV) reaches AT value, the node turns on its transmitter and transmits data to its $\mathrm{CH}$; otherwise, the node sets itself to idle and waits for the next slot. This is the HEEC protocol with AT (HEEC-AT).

Moreover, in order to meet the demands of sensed data screening and time-efficient, we transmit another threshold - comparative threshold (CT) to each node. In this algorithm, the $\mathrm{CV}$ value is saved in an internal variable called sensed value (SV). Only if CV value reaches AT value and the difference between $\mathrm{CV}$ and $\mathrm{SV}$ is not less than CT value, the node transmits data to $\mathrm{CH}$; otherwise, the node sets itself to idle and waits for the next slot. This is the HEEC protocol with CT (HEEC-CT).

\section{SIMULATION}

We have implemented our protocol with TEEN and DDEEC protocols to evaluate the performance based on the energy consumption and data packet transmission numbers.

\section{A. Simulation Environment.}

For our experiments, we set up a varying humidity environment which refers to Relative Humidity $(\mathrm{RH})$ in different regions particularly. Without any loss of generalization, we assume the sensing area is a square with a bound of $100 \mathrm{~m}$ and all 100 nodes are randomly distributed in the area and a BS is located in the center of the area. Similar with DDEEC protocol, we ignore the effect caused by signal collision and interference in the wireless channel. The radio parameters used in the simulations are shown in Table I. Furthermore, we assume the region of sensed data is from 0 to 100 and the values of AT and CT are 50 and 1 respectively.

In these experiments, we focus on three parameters: a) the time space from the network starts its operation until the death of first node (Stability Period), b) the time space between the network starts and the last node death (Network Lifetime) and c) the number of data packets received by base station.

TABLE I. PARAMETERS USED IN SIMULATIONS

\begin{tabular}{|c|c|}
\hline Parameters & Values \\
\hline$E_{\text {elec }}$ & $50(\mathrm{~nJ} / \mathrm{bit})$ \\
\hline$\varepsilon_{f s}$ & $10\left(\mathrm{pJ} / \mathrm{bit} / \mathrm{m}^{2}\right)$ \\
\hline$\varepsilon_{m p}$ & $0.0013\left(\mathrm{pJ} / \mathrm{bit} / \mathrm{m}^{4}\right)$ \\
\hline$E_{0}$ & $0.5(\mathrm{~J})$ \\
\hline$E_{D A}$ & $5(\mathrm{~nJ} / \mathrm{bit} / \mathrm{signal})$ \\
\hline$d_{0}$ & $70(\mathrm{~m})$ \\
\hline$N$ & 100 \\
\hline Message & $4000(\mathrm{bits})$ \\
\hline
\end{tabular}

\section{B. Simulation Results.}

In this section, we provide the simulation results of HEER-AT, HEER-CT, TEEN, and DDEEC under two situations in heterogeneous environments. We doesn't take into account the packets transmitted from normal nodes to sink directly, due to whose data cannot reflect the true information of monitoring environment and not suit for practical applications.

1) $m=0.2 \& a=3$ : The initial energy of normal nodes are $0.5[\mathrm{~J}]$ with the probability of 0.8 and of advanced nodes are $4 * 0.5=2[\mathrm{~J}]$ with the probability of 0.2 . Stability period is one of the most significant parameters because that almost all application users want to know the sensed data of the region where nodes were deployed. In order to ensure this, we have to make sure that all the sensors or at least almost sensors are alive. Fig. 2 presents the number of nodes those remain alive through the simulation period and it is obvious that our proposed protocol HEEC performance more efficient than TEEN and DDEEC both under Stability Period and Network Lifetime. HEEC-AT achieves the longer stability period by $78 \%$ than TEEN and $80 \%$ than DDEEC. HEEC-CT performance even better and prolong the stability period about $23 \%$ than HEEC-AT. From Fig. 2, we can obviously know the network lifetime of HEEC is much longer than that of DDEEC either. The number of nodes those are alive in TEEN is always 20 through round 2000 to 7300, which is exactly in line with the probability of advanced nodes $20 \%$. The curve of network lifetime for TEEN cannot show its better performance, but show that it is not suit for heterogeneous networks and cannot well balance the energy comsuption in the whole network.

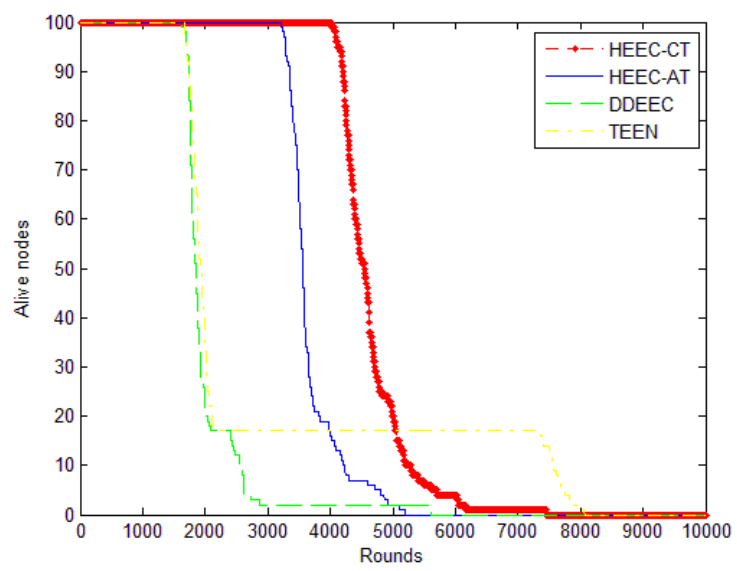

Figure 2. Alive Nodes $(\mathrm{m}=0.2 \& \mathrm{a}=3)$. 


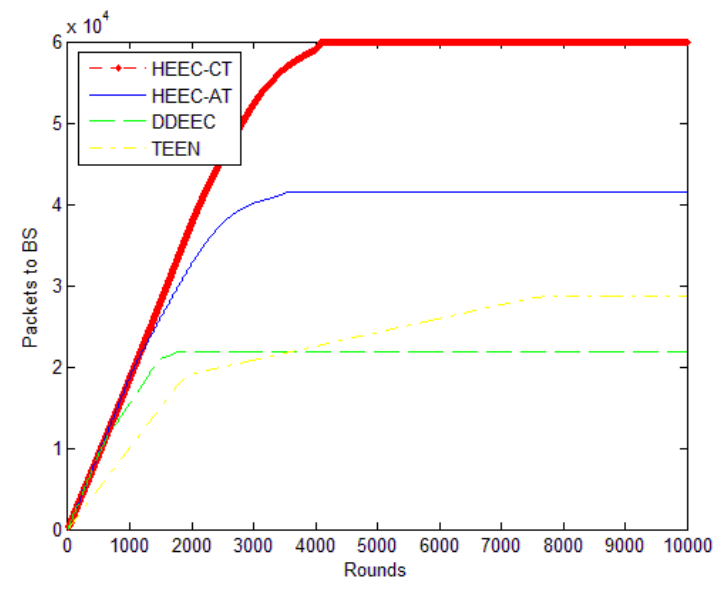

Figure 3. Packets to Base Station ( $\mathrm{m}=0.2$ \& $\mathrm{a}=3)$.

The amount of data packets received by BS decides how much information the users will get from the wireless sensors networks. So the more data packets received, the more efficient the protocol is. Fig. 3 shows that HEEC delivers more data to BS than TEEN or DDEEC.

2) $m=0.1 \& a=5$ : Fig. 4 presents the number of nodes those remain alive under heterogeneous environment. It is obvious that HEEC performance much better than TEEN and DDEEC. HEEC-AT can prolong the stability period about by $65 \%$ than TEEN and $86 \%$ than DDEEC. HEECCT prolong the stability period about $28 \%$ than HEEC-AT. Although TEEN has the long network lifetime, the amount of data packet received by the BS is much less than others in stability period which is shown in Fig. 5 . Fig. 5 also shows that the base station received more sensed data packets from HEEC than TEEN and DDEEC.

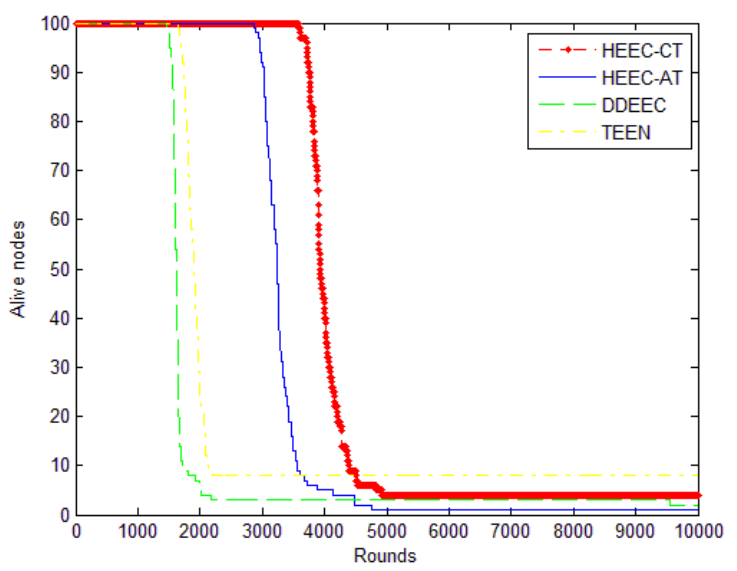

Figure 4. Alive Nodes ( $\mathrm{m}=0.1 \& \mathrm{a}=5)$.

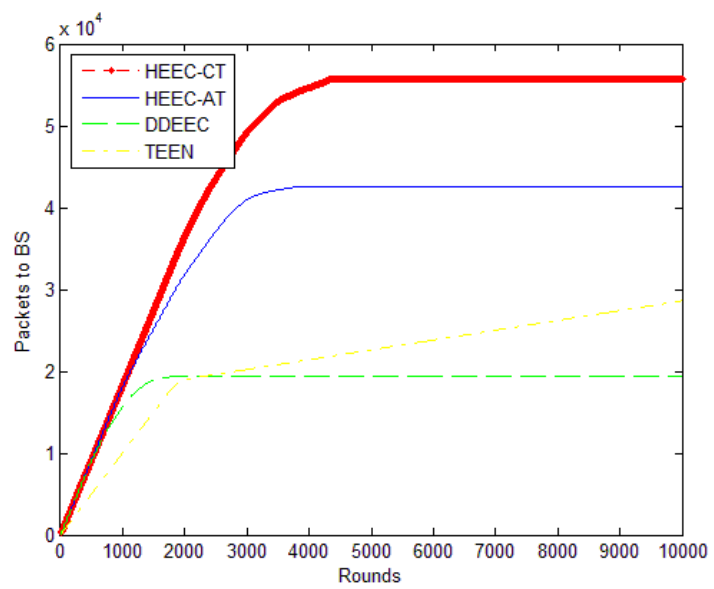

Figure 5. Packets to Base Station $(\mathrm{m}=0.1 \mathrm{\&} a=5)$.

The comparison of simulation results is shown in Table II below, which shows that our proposed protocol HEEC performance more efficient and performance than TEEN and DDEEC both under stability period and packets.

TABLE II. COMPARISON OF SIMULATION RESULTS

\begin{tabular}{|c|c|c|c|c|}
\hline \multicolumn{2}{|c|}{} & First Node Die & All Nodes Die & Packets \\
\hline \multirow{3}{*}{1} & TEEN & 1805 & 8006 & 28756 \\
\cline { 2 - 5 } & DDEEC & 1793 & 2980 & 22015 \\
\cline { 2 - 5 } & HEEC-AT & 3216 & 5128 & 41704 \\
\cline { 2 - 5 } & HEEC-CT & 3945 & 6115 & 59985 \\
\hline \multirow{2}{*}{2} & TEEN & 1750 & $>10000$ & 29005 \\
\cline { 2 - 5 } & DDEEC & 1556 & 1995 & 19956 \\
\cline { 2 - 5 } & HEEC-AT & 2895 & 4800 & 42823 \\
\cline { 2 - 5 } & HEEC-CT & 3705 & 4905 & 57085 \\
\hline
\end{tabular}

\section{CONCLUSION}

We have illustrated HEEC protocol that is well suitable for time critical applications, especially for military application areas in WSNs. Results from our experiments show that HEEC retains the advantages of TEEN and DDEEC and provides the better performance and more efficient energy conservation under wireless sensor networks.

\section{REFERENCES}

[1] Amiya Nayak and Ivan Stojmenovic, Wireless Sensor and Actuator Networks Algorithms and Protocols for Scalable Coordination and Data Communication, John Wiley \&Son Inc., 2010, pp. 1-16.

[2] Ian F. Akyildiz and Mehmet Can Vura, Wireless Sensor Networks, John Wiley \&Son Inc., 2010, pp. 17-33.

[3] W. Rabiner Heinzelman and H. Balakrishnan, "Energy-efficient communication protocol for wireless microsensor networks”, IEEE, 
Proceeding of the 3rd Hawali International Conference on System Science, 2000.

[4] W. Heinzelman, A. Chandrakasan, and H. Balakrishnan, "An application specific protocol architecture for wireless microsensor networks", IEEE Transactions on Wireless Communications, 2002.

[5] L. Qing, Q. Zhu, M. Wang, "Design of a distributed energy-efficient clustering algorithm for heterogeneous wireless sensor networks", In ELSEVIER, Computer Communications, 2006.

[6] Elbhiri B. and R. Saadane, "Developed distributed energy-efficient clustering (DDEEC) for heterogeneous wireless sensor networks", In IEEE I/V Communications and Mobile Network (ISVC), 2010.
[7] Sanjee Kumar Gupta, Neeraj Jain and Poonam Sinha, "Clustering protocols in wireless sensor networks: a survey", International Journal of Applied Information Systems (IJAIS), 2013.

[8] Mustapha Reda Senouci, Abdelhamid Mellouk, Hadj Senouci and Amar Asissani, "Performance evaluation of network lifetime spatialtemporal distribution for WSN routing protocols", Journal of Netwrok and Computer Applications 35, 2012, pp.1317-1328.

[9] A. Manjeshwar and D. P. Agarwal, "TEEN: a routing protocol for enhanced efficiency in wireless sensor networks”, 1st International Networks and Mobile Computing, 2001. 